\title{
The Level of Expression of Ki-67 in Invasive Cervical Cancers and Cervical Intraepithelial Neoplasia in Ghanaian Women
}

\author{
Ama Afrah*, Yao Tettey and Richard Kwasi Gyasi \\ Department of Pathology, University of Ghana Medical School, Ghana \\ *Corresponding author: Ama Afrah, Department of Pathology, College of Health Sciences, University of Ghana Medical School, Ghana
}

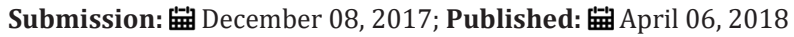

\begin{abstract}
Background: Increased cellular proliferation and cell cycle abnormalities have been associated with development of cervical cancer and cervical intraepithelial neoplasia in women. Criteria for grading of these lesions by pathologist based on digression in growth and degree of invasiveness among other abnormalities have been affected by inter and intra-observer differences resulting in poor reproducibility. Ki-67, a proliferative marker is useful in grading of cervical cancer and cervical intraepithelial neoplasia by giving uniform and reliable outcome independent of inter and intra-observer differences.
\end{abstract}

Aim: To demonstrate the level of expression of Ki-67 antigen in invasive cervical cancer and cervical intraepithelial neoplasia in Ghanaian women

Methodology: Using indirect immunohistochemical method, 116 diagnostic cervical samples with varying grades of cervical intraepithelial neoplasia and invasive cancer selected retrospectively and randomly was analyzed for level of expression of Ki-67.

Results: The levels of Ki-67 expression in malignant lesions were higher than in premalignant lesions which were also higher than in normal cervix. The levels of Ki-67 could distinguish post menopausal atrophy from dysplasia.

Conclusion: Due to semi quantitation of Ki-67 protein there exist some level of inter observer difference using Ki-67 grading of tumours but as compared to that which exist for histomorphological grading of tumours, the former is better. Inter observer difference using Ki-67 grading as compared to histomorphological grading of tumours were better using kappa analysis. Ki-67 score can distinguish between reactive lesions and dysplasia. Ki-67 analysis therefore should serve compliment to histological grading of tumour for the objective, reproducible, and reliable classification of dysplastic changes in cervical epithelium especially. However, the findings and conclusions of this study are limited by the small sample size of participants, and a much larger population-based study would be required to validate our findings.

Abbreviations: CIN: Cervical Intraepithelial Neoplasia; PD: Poorly Differentiated; MD: Moderately Differentiated; WD: Well Differentiated; INV: Invasive; HG: High Grade; LG: Low Grade; CIS: Carcinoma in Situ; ACSUS: Atypical Squamous Cell of Undetermined Significance

\section{Introduction}

Increased cellular proliferation and cell cycle abnormalities have been associated with premalignant and malignant lesions and for that matter cervical intraepithelial neoplasia (CIN) and invasive cervical cancer respectively [1]. Histological assessments which was the gold standard of increased cellular proliferation in the cervix by pathologists use the location and numbers of abnormal mitotic figures in the diagnosis of CIN and invasive cervical cancer [2]. Criteria for grading of these cervical lesions is based on deviation from the normal cell such as varying nuclear size and shape, hyperchromasia, angulations of nucleus, coarse chromatin, digression in rate of growth, degree of invasiveness, among other abnormalities [3].

Grading of these lesions among pathologists however have always been affected by inter and intra-observer differences so far as reproducibility assessment is concerned [4]. Due to inter and intra-observer differences in grading of epithelial lesions of especially the uterine cervix, use of adjutant methods such as radio labeling with ['H] thymidine and bromodeoxuridine have been considered $[5,6]$. Although quantitation of the proliferation rate of cellular deoxyribonucleic acid (DNA) can be assessed with some of these adjutant methods, their use in routine practice is limited due to sophistication. They require either in vitro or in vivo infiltration of tissue prior to fixation or access to specialized and expensive imaging equipment [7].

Ki-67 is a human non-histone nuclear protein, the expression of which is sternly associated with cell proliferation and which is widely used in routine pathology as a proliferation marker to measure the growth fraction of cells in human tumours [8]. 


\section{Study Design}

\section{Study design}

This was retrospective study. Archived cervical samples were selected using stratified random sampling from year 2007-2010 cases and analyzed immune histochemically for Ki-67 positivity in frank cervical cancers and CINs.

\section{Study site}

The study was done in the Department of Pathology, University of Ghana Medical School (UGMS), Korle -Bu Teaching Hospital (KBTH). The department has four main units; Histology, Cytology, Immunology and mortuary. The department undertakes a lot of clinical research especially in cancers and immunology.

\section{Subjects/target population}

The study population was from cervical specimen of women who have been diagnosed with invasive cervical cancer or CIN. Control population was from cervical samples of women negative for invasive cervical cancer or CIN from the archival samples in the Department of Pathology, UGMS, from year 2007 to 2010. Controls were matched against the cases according to age. Intensity of Ki67 expression among grades of CIN and invasive cancer was also compared.

\section{Inclusion Criteria}

\section{Study Cases}

Cervical Specimen of patients diagnosed with invasive cervical cancer or CIN aged between 25 to 90 years.

\section{Controls}

Cervical Specimen of patients negative for invasive cervical cancer or CIN aged between 25-90 years.

Exclusion criteria: Cases where paraffin embedded blocks is not available or tissue is depleted as well as cervical biopsies of women who fall outside the age range (25-90 years) diagnosed with or without cervical cancer.

Sample size: Using the prevalence rate of cervical cancer at $10.80 \%$ (estimated) obtained from 2010 annual report from the Department of Pathology University of Ghana Medical School, Korle$\mathrm{Bu}$. in Ghana the sample size was calculated from the formula: $\mathrm{n}=\mathrm{z} 2$ $(p, q) /($ error2), where $\mathrm{n}$ is sample size, $\mathrm{z}$ is standard-score at $95 \%$ confidence interval $=1.96, p$ is the prevalence of cervical neoplasia $(10.8 \%)$, q is percentage of non -cervical neoplasia cases $=1-p$ and error $=2 \%$ (Significant difference).

$$
\begin{aligned}
& \mathrm{n}=\mathrm{z} 2(\mathrm{p}, \mathrm{q}) /(\text { error2) } \\
& \mathrm{n}=1.962 \quad(0.108, \quad 1-0 . \quad 0.108) /(0.0052)=3.8416(0 \\
& 0.108,0.892) / 0.0025=3.8416(0.0 .0963) / 0.0025 \\
& \mathrm{n}=148.03 \equiv 148.0
\end{aligned}
$$

Informed consent: Since the study is retrospective using archival materials and direct patient contact is not involved, consent was sought from the Head of Department of Pathology, University of Ghana Medical School

Sample preparation: Selected paraffin embedded blocks of test and controls were put on ice for 30 minutes. Using the microtome at a predetermined gauge of $5 \mu \mathrm{m}, 3$ sections each were cut and allowed to float on water in a bath at a temperature of 600 $\mathrm{C}$ (slightly above the melting point of wax) to spread the folds in the sections but not melting the wax. Sections were put on slide and labeled with the research code and allowed for the water to drain. Pre-coated slides were used for immune section. Sections were put in oven subsequently for adhesion to slides. One slide of each patient and control was stained with haematoxylin and eosin (H\&E) to confirm diagnosis by a panel of reviewers and 1 of the remaining 2 slides was for immunostaining for Ki-67, the other slide was reserved in case there is a need for a repeat.

Staining and mounting haematoxylin \& eosin staining technique: After dewaxing in 3 changes of xylene for 2 minutes each, the sections were hydrated for 2 minutes each in absolute ethanol through descending grades of ethanol to water. The nuclei in the sections were then stained for 5 minutes in aqueous haematoxylin and afterwards blued using bluing agent at $\mathrm{pH}$ 7.0. Blued sections are dehydrated through ascending grades of ethanol to absolute for 2 minutes. Eosin, a counterstain was used to stain the cytoplasm of the cells in the sections. After counterstaining the sections were further dehydrated in three changes of absolute ethanol and removal of excess Eosin as well. Using 2 changes of Xylene, sections were cleared of ethanol and also allowed cytoplasmic transparency for 2 minutes and mounted using (DPX) mountant and coverslip appropriately.

Procedure for immunohistochemical staining: Using direct Immunohistochemical Staining Method based on the principle that when foreign antigens are introduced into a host, corresponding antibodies are produced was used to demonstrate ki-67. A primary antibody binds to the epitope FKEL to form an antigen-primary antibody complex. Antigen-primary antibody complex gave colour after an enhancer was added.

Paraffin sections were dewaxed and incubated in deionized water (AD) for 5 minutes. Usingretrieval solution antigen were retrieved ( 1 retrieval solution: 9 deionized water) at $99^{\circ} \mathrm{C}$ for 10 minutes. After the temperature was cooled to $50{ }^{\circ} \mathrm{C}$ the sections were equilibrated at room temperature in deionzed water to stop the antigen retrieval for 5 minutes. Epitope apart from the one to be investigated (FKEL) were blocked to prevent non-specific binding using $200 \mu \mathrm{l}$ of perioxidase for 5 minutes. With the exception of the test negative controls and negative controls each section was treated with $200 \mu$ l of prediluted (dilution factor not disclosed by manufacturer) primary antibody by incubation at room temperature for 30 minutes to bind to corresponding antigen. After that $200 \mu \mathrm{l}$ of visualization reagent horse radish perioxidase (HRP) was applied to each section for 15 minutes. $200 \mu$ l of AP, a second visualizing reagent was further applied and incubated for 15 minutes. $200 \mu \mathrm{l}$ of DAB substrate-chromogen (1ml DAB substrate: $30 \mu \mathrm{l}$ of DAB 
chromogen; stable within 8 hours of dilution) was later applied to each section and incubated for 10 minutes. Afterwards each section was flooded with deionised water to stop the reaction of DAB substrate-chromogen for 5 minutes. $200 \mu \mathrm{l}$ of Fast Red substratechromogen (1ml Fast Red substrate: $45 \mathrm{ml}$ Fast Red chromogen) was applied to each section for an incubation period of 15 minutes. The sections were finally flooded with $2 \mathrm{ml}$ of distilled water for 5 minutes. After each major step stated above, each section were flooded with $2 \mathrm{ml}$ of wash buffer ( 1 wash buffer: 9 deionized water) and incubated for 5 minutes to remove excess reagent. All steps were performed at room temperature and sections were washed in phosphate buffered saline. Counter staining with Haematoxylin as in HE was done as above. Slides were mounted using DPX.

\section{Microscopy}

The H\&E stainedsections were light microscopically examined using x10, x25 and x40 objectives of Olympus microscope, CX41RF.
X10 objective was for scanning through the section and $\mathrm{x} 40$ objectives for detailed evaluation to confirm diagnosis. The sections of test cases was compared with controls.

\section{Immunostained sections}

Evaluation of the immunohistochemistry was done by light microscope as well. The immune-stained sections were confirmed by 2 Reviewers using $\mathrm{x} 10$ and $\mathrm{x} 40$ objectives of Olympus microscope CX41RF using $\mathrm{x} 10$ objective to scan through the smear and $\mathrm{x} 40$ objectives for detailed evaluation of Ki-67 expression. The sections of test cases was compared with controls. The grading was done as follows: $0-20 \%$ as normal, $21-34 \%$ as LG; $35-66 \%$ as MG, $67-70 \%$ as $\mathrm{HG}$, and above $70 \%$ as INV.

\section{Results}

Tables 1-4 and Figure 1.

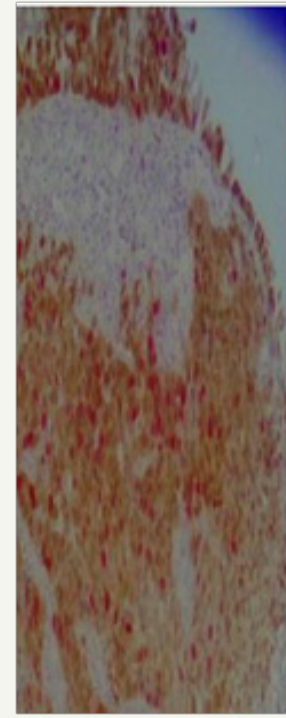

A1

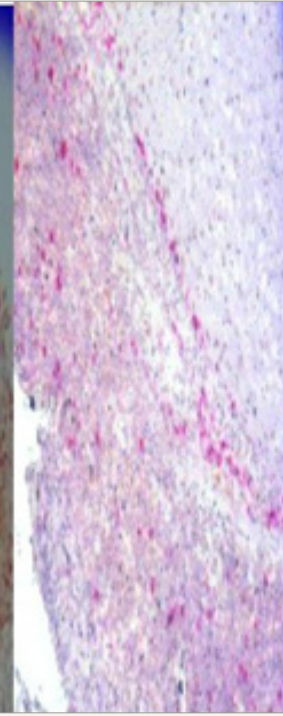

A2

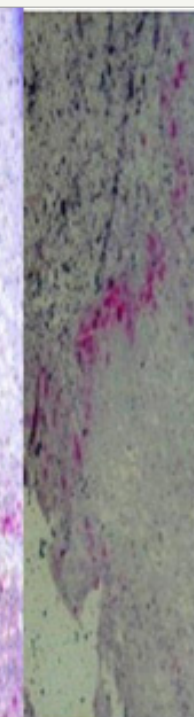

A3

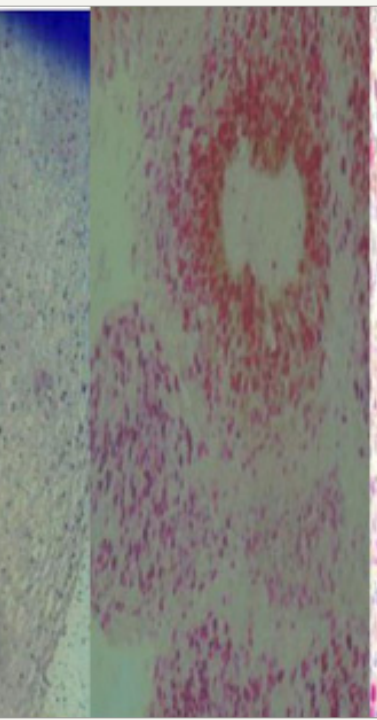

A4

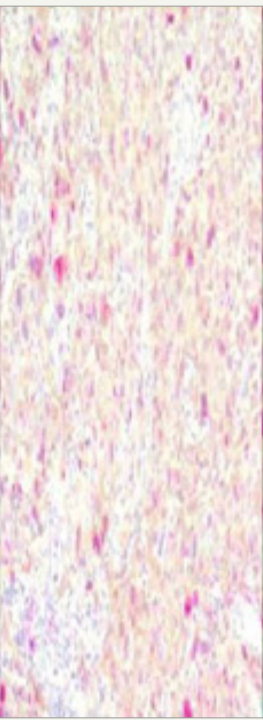

A5

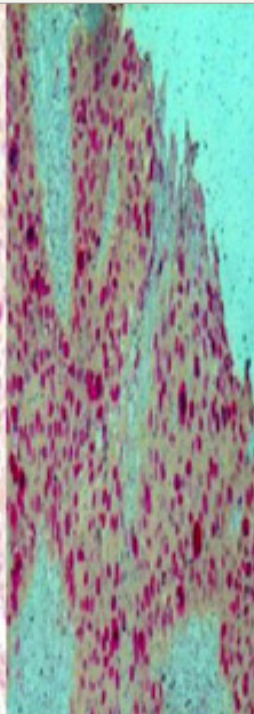

A6

Figure 1: Micrographs of Ki-67 stained section (x 40).

Figure A1: Control positive expressing 60\% Ki-67 which may be due HPV infection showing no cytopathic effect.

Figure A2: HPV about $30 \%$ correlates with diagnosis.

Figure A3: CIN II expressing Ki-67 up to 25\%.

Figure A4: Well Differentiated carcinoma Ki- expressing Ki-67 up to $>70 \%$ Figure A5 : Carcinima In Situ expressing Ki-67 up to $70 \%$.

Figure A6: Invasive Squamous Cell Crcinoma expressing about $>70 \%$ of Ki-67.

Table 1: Table of histomorphological diagnosis in study ‘population.

\begin{tabular}{|c|c|}
\hline Type of SCC/CIN & Quantity \\
\hline Invasive & 19 \\
\hline WD & 13 \\
\hline MD & 38 \\
\hline PD & 18 \\
\hline CIS & 11 \\
\hline
\end{tabular}

\begin{tabular}{|c|c|}
\hline CIN III & 6 \\
\hline CIN II & 2 \\
\hline CIN I & 1 \\
\hline Koilocytes & 6 \\
\hline ASCUS & 1 \\
\hline Moderate/Poor & 1 \\
\hline Total & 116 \\
\hline
\end{tabular}


Table 2: Level of expression of ki -67 in premalignant cervical lesions.

\begin{tabular}{|c|c|c|c|c|c|c|c|}
\hline Percentage of Ki-67 (\%) & $\mathbf{0}$ & $\mathbf{5 2 0}$ & $\mathbf{2 1 - 3 4}$ & $\mathbf{3 5 - 6 6}$ & $\mathbf{6 7 - 7 0}$ & $\mathbf{2 7 0}$ & Total \\
\hline CIN I & 0 & 0 & 0 & 0 & 0 & 0 & 0 \\
\hline CIN II & & & 1 & 1 & 0 & - & 2 \\
\hline CIN III & 0 & 0 & 0 & 0 & 1 & 1 & 3 \\
\hline koilocytes/con doloma & & & 1 & & 0 & 0 & 1 \\
\hline ASCUS & & & 1 & 0 & 1 & 3 & 8 \\
\hline Total & & & 3 & 1 & 1 & & 0 \\
\hline
\end{tabular}

Table 3: Histological diagnosis with their corresponding level of ki-67 expressions malignant.

\begin{tabular}{|c|c|c|c|}
\hline Slide No & Histomorphological Diagnosis & Level of Ki -67 Expression \% & Coreespondi Ng Diagnosis \\
\hline 1 & WD 01 & - & - \\
\hline 2 & PD & NEG & INV \\
\hline 3 & WD 01 & $>70$ & HG \\
\hline 4 & CIS & $67-70$ & LG \\
\hline 5 & KOILOCYTES & INV & INV \\
\hline 6 & CIN III & $>70$ & INV \\
\hline 7 & MD & $>70$ & INV \\
\hline 8 & PD & $>70$ & INV \\
\hline 10 & WD & $>70$ & \\
\hline
\end{tabular}

Table 4: Kappa analysis for KI-6.

\begin{tabular}{|c|c|c|}
\hline Diagnosis & Ki-67 & Histomorphology \\
\hline WD & 0.95 & 0.361 \\
\hline MD & 0.82 & 0.606 \\
\hline PD & 0.72 & 0.136 \\
\hline CIS & 0.75 & 0.305 \\
\hline CIN III & 0.71 & 0.305 \\
\hline Koilocyte & 0.85 & 1 \\
\hline
\end{tabular}

\section{Discussion}

\section{Histomorphological diagnosis in study population}

Histomorphological diagnosis in the study population in the study population ranged from poorly differentiated Squamous cell Carcinoma to CIN I. Out of 116 diagnostic test cases the premalignant cases were 16 in number representing $13.80 \%$ whilst the malignant ones are 102 in number (86.10\%). For the malignant cases, Invasive moderately Differentiated Squamous Carcinoma (MD) numbering 38(32.76. \%) was most diagnosed whilst well differentiated squamous cell carcinoma (WD) was the 13(11.21\%) in number. $18(15.52 \%)$ were diagnosed as poorly differentiated squamous cell carcinoma (PD) whilst CIS the least diagnosed was $11(9.48 \%)$. There were $19(16.38 \%)$ invasive squamous cell carcinoma with differentiation. There were 6(5.17\%) CIN III, $2(1.72 \%)$ CIN II and 1 CIN I (0.86\%) Cervical cancer worldwide have attributed to HPV infection [9].

Cervical cancer remains the second most common cancer and leading cause of cancer deaths among women living in low to middle income countries and for that matter Ghana [10]. This trend of events indicates that African women and for that matter Ghanaian female (no published data) present late with cervical lesions to the hospital with subsequent poor prognosis for longterm survival [11]. Again poor viral clearing due to weak immune system as a result of few langerhan cells resulting in persistence of viral infected cervical cell in smokers which is rare in the case of Ghana have been investigated [12]. Most of these women live in deprived areas with very low socio-economic status as reported by Hannele et al. [13].

Hormone replacement therapy and oral contraceptive use which is inconclusive have been cited as cofactors to HPV infection in some studies [12]. A USA study found fourfold increase in risk of developing pre-invasive lesions overall in using hormonal contraceptive with risk increasing with duration of use [14]. In the developed world prescreening is done before to assess who is a good candidate for a particular method of contraception whiles in our case, this is not so [15].

Poor nutrition among women who may be subsequently infected with HR HPV develops cervical cancer as compared with infected women taking good nutrition [16]. Most of these women with cervical cancer may have had their first sexual debut in early ages with the risk increasing with number of life-time partners as stated by Akosua et al. [17]. As confirmed by some researches, high parity increases a women's chance of developing cervical cancer; the greater the number of children the higher the risk of developing cervical cancer with age at first birth been important [18]. 
The prevalence of cervical cancer in older women of this study confirms an earlier one which indicates that most women are in polygamous marriages where there is high prevalence of HPV in older age groups, which may be a distinctive feature of polygamous populations where HPV transmission continues into middle age and cervical cancer incidence is very high [17].

\section{Histomorphological diagnosis with their corresponding level of ki-67 expression}

Histological review and corresponding Ki-67 were scored independently by the same pathologist and the result is as follows. The level of expression of Ki-67 in 3 (30\%) originally diagnosed WD SCC and reviewed as 2(20\%) WD and 1 (10\%) MD were $>70 \%$ corresponding to INV according to the reference table of this study (Table 4)

The level of expression of Ki-67 in one case originally diagnosed as PD SCC was negative (0\%) by R 1 but for some unexplained reason did not review its diagnosis. This $0 \%$ expression of Ki-67 could be due to misdiagnosis originally which could be due to reactive changes or atrophy which Ki-67 is said to have distinguished between the two. Both atrophy and CINs show increased nuclear/ cytoplasmic ratio, nuclear crowding, and lack of maturation. Due to these similarities, distinguishing CIN from atrophy in cervical biopsies from elderly patients is often controversial. Unlike atrophy, dysplasia show increased cellular proliferation. Hence the possiblility of utilizing of KI-67 in distinguishing dysplasia from atrophy [19]. This is supported of a study done in the Netherlands in which using only histomorphology in diagnosis cervical atrophy was classified as HG lesion but level of expression of Ki-67 which was lower than in nomal cervix confirmed it to be atrophy but not dysplasia as suggested by morphological diagnosis [20].

The level of expression of Ki-67 in a reviewed WD originally CIN III was also $>70 \%$ correlating with INV. This support a research by Looi et al. [21] in which Ki-67 index was higher in high grade CIN (CIN II and III) and SCC lesions compared to normal cervices. Reproducibility of grading as WD, MD, PD, CIS, CIN III, Koilocytes by R1 using 'Ki-67 immunostaining (weighted Kappa- 0.95, $0.82,0.72,0.75,0.71$ and 0.85 respectively) were all higher than histomorphology (weighted Kappa- 0.361, 606, 0.136, 0.305, 0.305 and 1.000 respectively.). The average weighted Kappa using Ki67 immunostaining to grade is 0.80 . The average weighted Kappa using histomorphology to grade is 0.452

\section{Level of expression of ki -67 in premalignant cervical lesions}

$2(25 \%)$ of the study population in the premalignant (CIN II) category had 1(12.50\%) expressing Ki-67 level between 21-34 \% corresponding to LG whilst the other 1 expressed level Ki-67 between $35-66 \%$. 2 (25\%) CIN III had their level of expression of Ki-67 between 67-70 \% correlating to HG lesion. Out of 3(37.5\%) koilocytes/condoloma, $1(12.5 \%)$ had its level of expression of Ki-67 between 21-34 \% corresponding to LG, whilst the other
1(12.5\%) had its Ki-67 level between 35- $66 \%$ corresponding to HG. $1(12.5 \%)$ of ASCUS had ki-67 expressed between 21-34\% corresponding to LG lesion. Dependent T-test was used to compare the number of cases classified by KI-67 and manual diagnosis. No significant difference was found between the two diagnosis criteria $(\mathrm{t}=2.343, \mathrm{p}=0.167)$.

Premalignant cervical lesions have being attributed to HPV infection which after malignant transformation of cell there is increased in cellular proliferation which is positively correlated with Ki-67, a proliferative marker. The overall expression level of Ki-67 increased with higher grades of the lesion. The deviation in its expression can be attributed to inter observer differences in grading of the lesion. This research is in support of most studies done elsewhere. In a study by Mica et al. [22] Ki-67 immunostaining proved to be predictive for high-risk HPV infection, and could differentiate reactive lesions from cervical dysplasias. Again Ki-67 quantitative analysis in 3 epithelial layers was sensitive and specific method of differentiation between CIN 1, CIN 2 and CIN 3 grades and could be a adjunctive method for more accurate CIN grading. A Serbian research revealed that there was positive correlation between proliferative activity, distribution of Ki-67 positive cells and increasing CIN grade [23].

In contrast to this, a Swedish study revealed higher MIB-1 (KI 67) levels in tumors with a lower grade. Moreover, MIB-1 levels seem to be higher in tumors due to infection with HPV 16 and 18. It was concluded that MIB-1(Ki-67) may be a helpful marker in grading carcinoma: a high level of expression of MIB-1 indicates a low grade of tumor [24].

\section{Level of expression of ki -67 in malignant cervical lesions}

Out of the $19(19.56 \%)$ cases diagnosed histomorphologically as PD, Ki-67 could confirm only 12 (13.04\%) as invasive; $12(13.04 \%)$ their level of expression of Ki-67 greater than 70\%, 4(4.35\%) of PD had their level of expression of Ki-67 between 35-66\% whilst $3(3.26 \%)$ had their level between $21-34 \%$. Out of $38(41.30 \%)$ diagnosed histomorphologically as MD 12 (13.04\%) was picked by Ki-67 expression as such whilst the level of expression of Ki-67 in 6 (6.52\%) were $67-70 \%$ correlating with HG, the level of expression of $\mathrm{Ki}-67$ in $10(10.86 \%)$ was between $35-66 \%$ corresponding to $\mathrm{M}$, in $3(3.26 \%)$ the level was between $21-34 \%$ correlating to LG and $5(5.43 \%)$ had their expression $>20$ where research suggest it to be negative [25]. Out of $14(15.22 \%)$ histomorphologically cases of WD 6(6.52\%) were picked as such by Ki-67 as such, 12(13.04\%) of the study population had their of expression of Ki- 67 protein less than $20 \%, 2(2.17 \%)$ had Ki-67 protein expressed within $67-70 \%$ correlating to HG, $2(2.17 \%)$ had $\mathrm{Ki}-67$ protein expressed within $35-66 \%$ correlating to MD 3(3.26\%) had Ki-67 protein expressed within $21-34 \%$ correlating to LG.

18(19.57\%) were diagnosed as INV without any differentiation using histology; $10(10.87 \%)$ had Ki-67 expression above $70 \%$ correlating to INV, 4(4.35\%) had Ki-67 expression between 
35-66\% correlating to $\mathrm{HG}$ whilst another 4(4.35\%) had Ki-67 expression between $21-35 \%$ correlating LG. 4(4.35\%) of the study population was diagnosed as CIS histomorphologically all had their level expression of Ki-67>70\% correlating to INV. This support a research by Haas et al. [26] in which Ki-67 index was higher in SCC lesions compared to normal cervices. All cases of INV that expressed low levels of Ki-67 could be in actual fact HG that are regressing as suggested by a study Iaconis et al. [27].

\section{Conclusion}

Inter observer difference using histomorphological grading of tumours between seniors colleagues is greater as compared to juniors colleagues. This implies that the level of agreement between junior colleagues is higher than between senior colleagues.

Due to semi quantitation Ki-67 protein there exist some level of Inter observer difference using Ki-67 to compliment grading of tumours but as compared to that which exist for histomorphological grading of tumours, the former is better. As was observed in histomorphological diagnosis of especially lower grade lesions which was originally diagnosed Koilocytes was reviewed and diagnosis came out as benign, Ki-67 score could distinguish between reactive lesions and dysplasia. These inter observer and intra observer differences will affect patient management; under treatment which may result in cancer metastasis or over treatment which incur unnecessary cost on poor patients.

Ki-67 analysis is a promising alternative method for the objective, reproducible, and reliable classification of dysplastic changes in cervical epithelium especially using computerized image analysis system. However, the findings and conclusions of this study are limited by the small sample size of participants, and a much larger population-based study would be required to validate our findings.

\section{Acknowledgement}

I would like to give special thanks to CINTec, Professor R. K. Gyasi and Professor Y. Tettey my supervisors for doing me the honour to work with them. Their help guidance and help are beyond measure, God richly bless them.

\section{Appendix}

\section{Preparation of reagent - CINtec PLUS kits}

The reagent were all commercially prepared and some prediluted without dilution factor. The pack of kits contained, Peroxidase Blocking Reagent, MIB -1 primary antibody, a APvisualizing reagent, CIN mountant and HRP were all ready to use (Prediluted). Wash Buffer, DAB Buffered Substrate and Chromogen, Naphthol Phosphate Substrate and Fast Red Chromogen, Retrieval Solution were not diluted. Storage of kits was done at $5{ }^{\circ} \mathrm{C}$.

\section{Dilutions}

a. Wash Buffer-1 Part of Stock Buffer: 9 Parts Of AD.

b. Retrieval Solution -1 Part of Stock Buffer: 9 Parts of AD. c. DAB Buffered Substrate and Chromogen-016ML DAB Buffered Substrate: 0.85ML Chromogen Fast Red-25ML Naphthol Phosphate Substrate: 1.33 ML Fast Red Chromogen,

\section{References}

1. Preston-Martin S, Pike MC, Ross RK, Jones PA, Henderson B (1990) Increased Cell Division as a Cause of Human Cancer1; Cancer Res 50(23): 7415-7421.

2. Kalof AN, Cooper K (2007) Our approach to squamous intraepithelial lesions of the uterine cervix. J Clin Pathol 60(5): 449-455.

3. http://www.healthhype.com/cancer-staging-and-grading-stagesgrades-of-malignant-tumors.html

4. Fox H, Buckley CH (1990) Current problems in the pathology of intra-epithelial lesions of the uterine cervix. Histopathology 17 (1): 1-6.

5. Nicholas SA, Lin P, Morihara J, Mao M, Nancy BK, et al. (2003) p16INK4a expression correlates with degree of cervical neoplasia: A comparison with Ki-67 expression and detection of high-risk HPV types. Mod Pathol 16(7): 665-673.

6. Piroget al, 2002- Pirog, E.C., R.N. Baergen and R.A. Soslow, et al. Diagnostic accuracy of cervical low-grade squamous intraepithelial lesions is improved with MIB-1 immunostaining. Am. J.Surg. Pathol2002;26:7075

7. Svensson M, Mattsson P, Aldskogius H (1994) A bromodeoxyuridinelabelling study of proliferating cells in the brainstem following hypoglossal nerve transection. J Anat 185(3): 537-542.

8. Ancuța E, Ancuța C, Cozma LG, Iordache C, Anghelache-Lupaşcu I, et al. (2009) Tumor biomarkers in cervical cancer: focus on Ki-67 proliferation factor and E-cadherin expression. Rom J Morphol Embryol 50(3): 413-438

9. Burd EM (2013) Human papillomavirus and cervical cancer. Lancet 16(1): $1-17$.

10. Jemal A, Bray F, Center MM, Ferlay J, Ward E, et al. (2011) Global cancer statistics. CA Cancer J Clin 61(2): 69-90.

11. Abotchie PN, Shokar NK (2009) Cervical Cancer Screening Among College Students in Ghana: Knowledge and Health Beliefs. Int J Gynecol Cancer 19(3): 412-416.

12. Szarewski A, Jarvis MJ, Sasieni P, Anderson M, Edwards R, et al. (1996) Effect of smoking cessation on cervical lesion size. Lancet 347(9006): 941-943.

13. Hannele Palosuo, Seppo Koskinen, Eero Lahelma, Elisa Kostiainen, Ritva Prättälä, et al. (1995) Health inequalities in Finland trends in socioeconomic health differences1980 - 2005 ministry of Social Affairs and Health Publications p. 9

14. Schlesselman JC (1995) Net effect of oral contraceptive use on the risk of cancer in women in united states. Obstet Gynecol 85: 793-801.

15. www.womenhealth.com -may

16. http://www.canceractive.com/cancer-active-page-link.aspx?n=1243 $-18 / 5 / 15$

17. Domfeh A, Wiredu E, Adjei A, Ayeh-Kumi P, Adiku T, et al. (2008) Cervical Human Papillomavirus Infection in Accra, Ghana. Ghana Med J 42(2): 71-78.

18. http://www.cancerresearchuk.org/about-cancer/type/cervical-cancer/about/cervical-cancer-risks-and-causes -17/5/15

19. Mittal K, Mesia A, Demopoulos RI (1999) MIB-1 expression is useful in distinguishing dysplasia from atrophy in elderly women. Int J Gynecol Pathol 18(2): 122-124. 
20. Bulten, Schijf C, van der Laak JA, Wienk S, Poddighe PJ (2005) Decreased expression of Ki-67 in atrophic cervical epithelium of post-menopausal women. J Pathol 190(5): 545-553.

21. Looiet al in which Ki-67 index was higher in SCC lesions compared to normal cervices.

22. Mimica M, Tomić S, Kardum G, Hofman ID, Kaliterna V, et al. (2010) Ki-67 Quantitative Evaluation as a Marker of Cervical Intraepithelial Neoplasia and Human Papillomavirus Infection. Int J Gynecol Cancer 20(1): 116119.

23. Milana P, Tatjana (2006) Ki-67 expression in squamous intraepithelial lesions of the uterine cervix. Arch Oncol 14(1-2): 23-25.
24. Muller S, Flores-Staino C, Skyldberg B, Hellström AC, Johansson B, et al. (2008) Expression of p16INK4a and MIB-1 in relation to histopathology and HPV types in cervical adenocarcinoma 32(2): 333-340.

25. Galand P. Ki-67 immunostaining of normal human epidermis: Heidebrecht HJ Buck.

26. Heidebrecht HJ, Buck F, Haas K, Wacker HH, Parwaresch R (1996) Monoclonal antibodies Ki-S3 and Ki-S5 yield new data on the 'Ki-67' proteins. Cell Prolif 29(7): 413-425.

27. Kill IR (1996) Localisation of the Ki-67 antigen within the nucleolus: Evidence for a fibrillarin-deficient region of the dense fibrillar component. J Cell Sci 109(PT 6): 1253-1263.

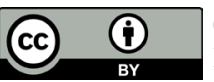

Creative Commons Attribution 4.0 International License

For possible submissions Click Here
Submit Article

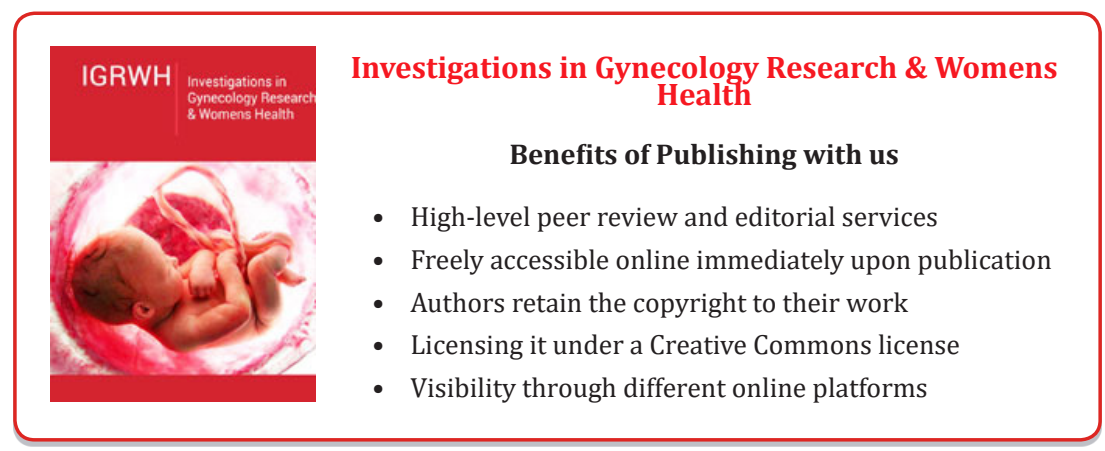

